\title{
Contributions to HiLiftPW-3 Using Structured, Overset Grid Methods
}

\author{
Presented at AIAA SciTech 2018 \\ Kissimmee, FL \\ January 10, 2018
}

\author{
Jim Coder \\ University of Tennessee, Knoxville
}

Tom Pulliam and James Jensen

NASA Ames Research Center 


\section{Outline}

- Introduction

- Description of HiLiftPW-3 Geometries and Cases

- Computational Methodologies

- Results

- Conclusion

- Acknowledgments 


\section{Introduction}

- Two geometries of interest

- High-Lift Common Research Model (HL-CRM)

- Completely predictive

- JAXA Standard Model (JSM)

- Transitional test case

- Structured, overset grids generated and provided by the organizing committee

- Two overset solvers considered in this paper

- OVERFLOW (UTK and NASA)

- LAVA (NASA) 


\section{Outline}

- Introduction

- Description of HiLiftPW-3 Geometries and Cases

- Computational Methodologies

- Results

- Conclusion

- Acknowledgments 


\section{HL-CRM Geometry}

- Open-source high-lift configuration based on the Common Research Model (Lacy and Sclafani, 2016)

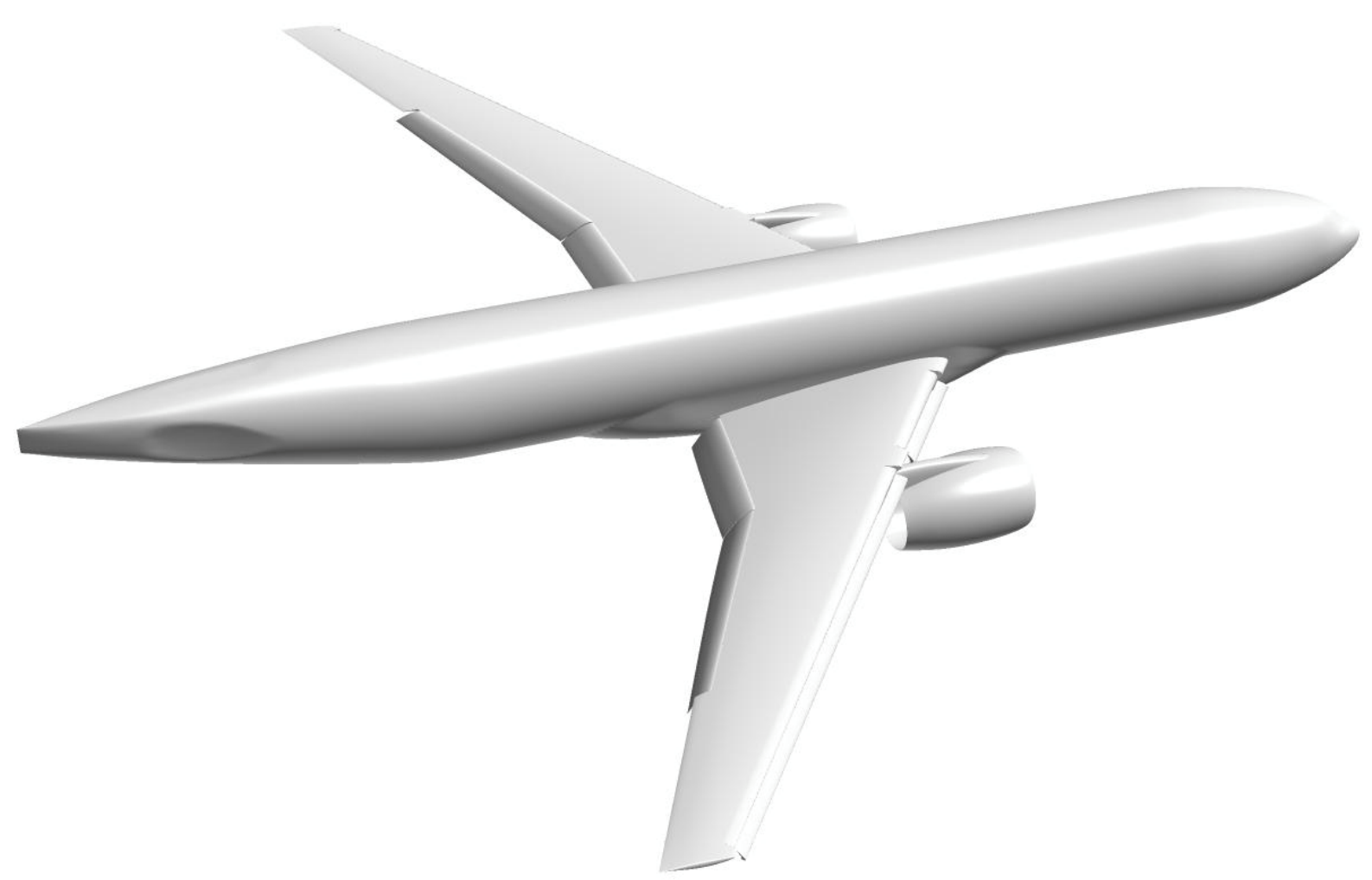




\section{HL-CRM Cases (Case 1)}

- Case 1a (requested): Full-Chord Flap Gap grid-refinement study

- Case 1b (optional): Full-Chord Flap Gap with grid adaptation

- Case 1c (optional): Partially Sealed Chord Flap Gap for medium-resolution grid only

- Case 1d (optional): Partially Sealed Chord Flap Gap with grid adaptation

\begin{tabular}{|l|l|}
\hline Free-stream Mach Number & 0.2 \\
\hline Angles of Attack & $8^{\circ}$ and $16^{\circ}$ \\
\hline Mean Aerodynamic Chord (MAC) & 275.8 in (full scale) \\
\hline Reynolds Number (based on MAC) & $3.26 \times 10^{6}$ \\
\hline Reference Static Temperature & $518.67^{\circ} \mathrm{R}(288.15 \mathrm{~K})$ \\
\hline Reference Static Pressure & $14.700 \mathrm{psi}(760.21 \mathrm{~mm}-\mathrm{Hg})$ \\
\hline
\end{tabular}




\section{JSM Geometry}

- Representative of a 100-person-class transport with a modern high-lift system (Yokokawa et al., 2006 and 2008)

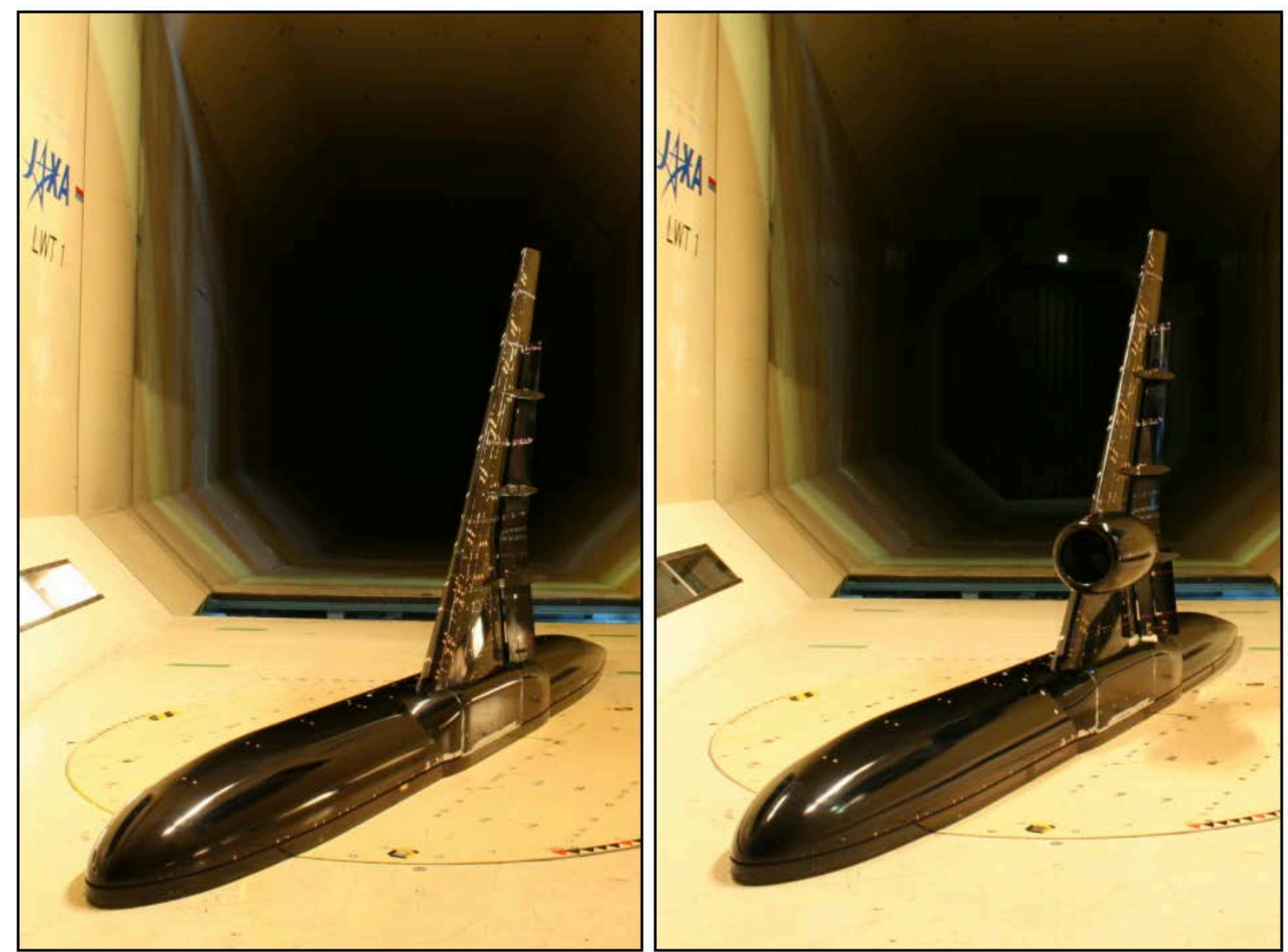




\section{JSM Cases (Case 2)}

- Case 2a (requested): Nacelle/Pylon Off

- Case 2b (optional): Nacelle/Pylon Off with grid adaptation

- Case 2c (requested): Nacelle/Pylon On

- Case 2d (optional): Nacelle/Pylon On with grid adaptation

\begin{tabular}{|l|l|}
\hline Free-stream Mach Number & 0.172 \\
\hline Angles of Attack & $4.36^{\circ}, 10.47^{\circ}, 14.54^{\circ}, 18.58^{\circ}, 20.59^{\circ}$, and $21.57^{\circ}$ \\
\hline Mean Aerodynamic Chord (MAC) & $529.2 \mathrm{~mm}$ (model scale) \\
\hline Reynolds Number (based on MAC) & $1.93 \times 10^{\circ}$ \\
\hline Reference Static Temperature & $551.79^{\circ} \mathrm{R}(306.55 \mathrm{~K})$ \\
\hline Reference Static Pressure & $14.458 \mathrm{psi}(747.70 \mathrm{~mm}-\mathrm{Hg})$ \\
\hline
\end{tabular}




\section{Outline}

- Introduction

- Description of HiLiftPW-3 Geometries and Cases

- Computational Methodologies

- Results

- Conclusion

- Acknowledgments 


\section{Flow Solvers and Approach}

- OVERFLOW 2.2 (UTK and NASA)

- Node-centered, finite-difference

- RHS discretization: $3^{\text {rd }}$-order MUSCL w/ Roe fluxes

- LHS algorithm: ARC3D scalar pentadiagonal solver

- Turbulence model: Spalart-Allmaras SA-noft2-RC-QCR2000

- Transition model: Coder AFT2017b (SA-RC-QCR2000-AFT2017b)

- Turbulence model variant and inclusion of transition modeling studied

- Time accuracy effects studied

- BDF2 implicit scheme

- Timestep chosen to give 2 orders of magnitude drop in unsteady residual in $10-20$ subiterations 


\section{Flow Solvers and Approach}

- LAVA (NASA)

- Node-centered, finite-difference

- RHS discretization: $2^{\text {nd }}$-order MUSCL w/ Roe fluxes

- Van Albada limiter

- Turbulence model: Spalart-Allmaras SA-noft2-RC-QCR2000

- "Cold starts" used for all cases 


\section{Computational Resources}

- All simulations run on NAS Pleiades

- SGIICE system

- Over 11,000 nodes with over 245,000 cores

- Intel Xeon (Broadwell, Haswell, Ivy Bridge, Sandy Bridge)

- OVERFLOW simulations run on 420 cores (fully turbulent) and 560 cores (transitional)

- 24-48 hours of wall-clock time to convergence

- LAVA required 2000 cores with 48 hours of wall clock time 


\section{Outline}

- Introduction

- Description of HiLiftPW-3 Geometries and Cases

- Computational Methodologies

- Results

- Conclusion

- Acknowledgments 


\section{Case 1: Surface Smoothness Issues}

- Original HL-CRM overset grids were projected onto a surface triangulation rather than the smooth CAD

- Leads to oscillatory pressure behavior

- New grids generated with projection directly to CAD

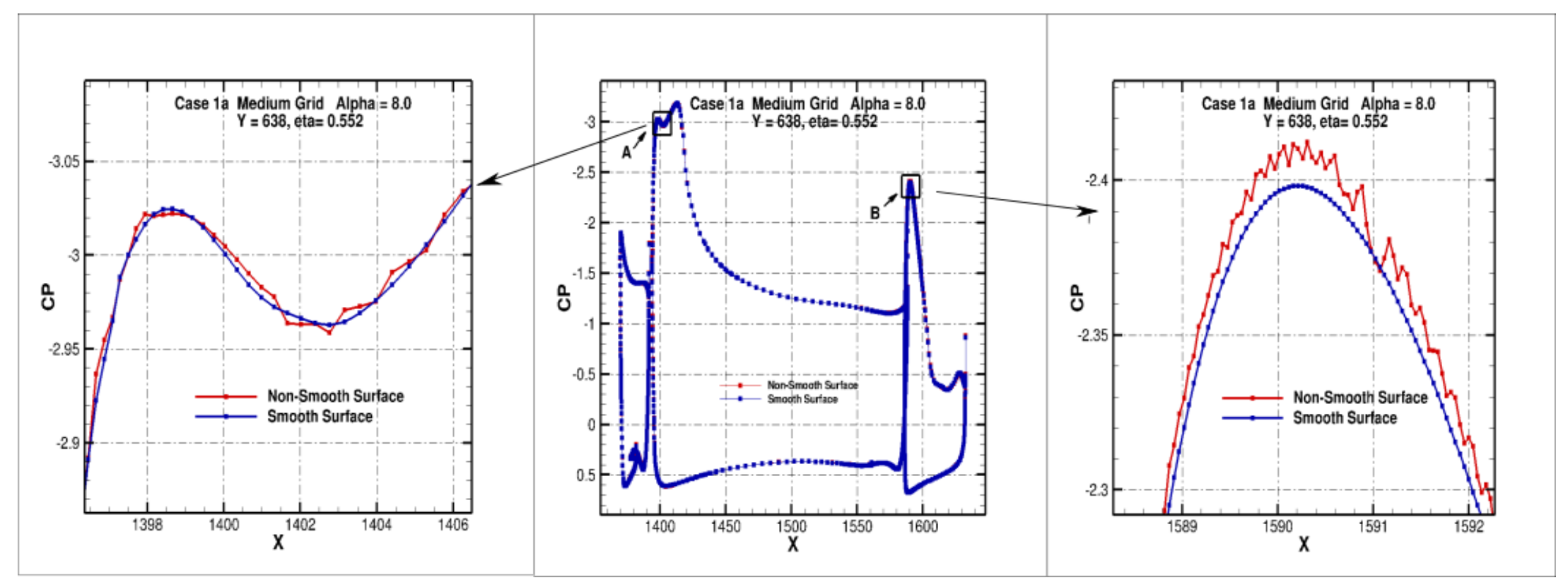




\section{Case 1: Turbulence Modeling Effects}

- Use (or exclusion) of QCR had a prominent effect on the flow behavior around the flap gap

- QCR typically regarded as primarily affecting juncture flows
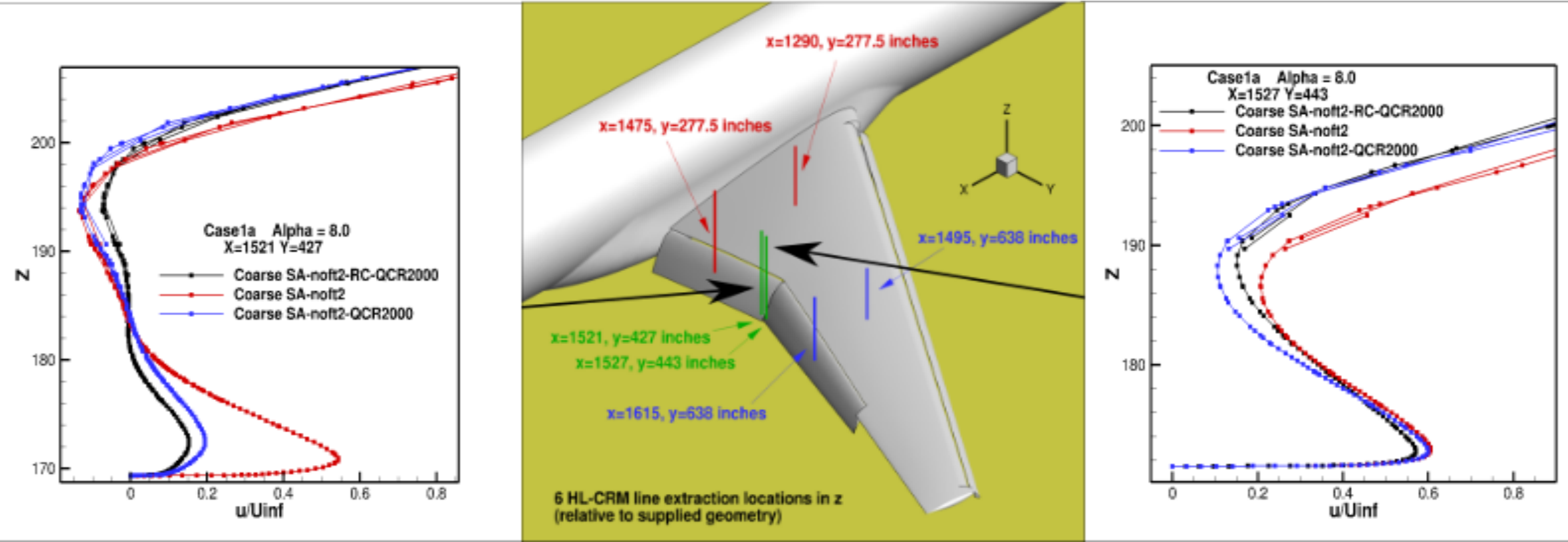


\section{Case 1: Turbulence Modeling Effects}

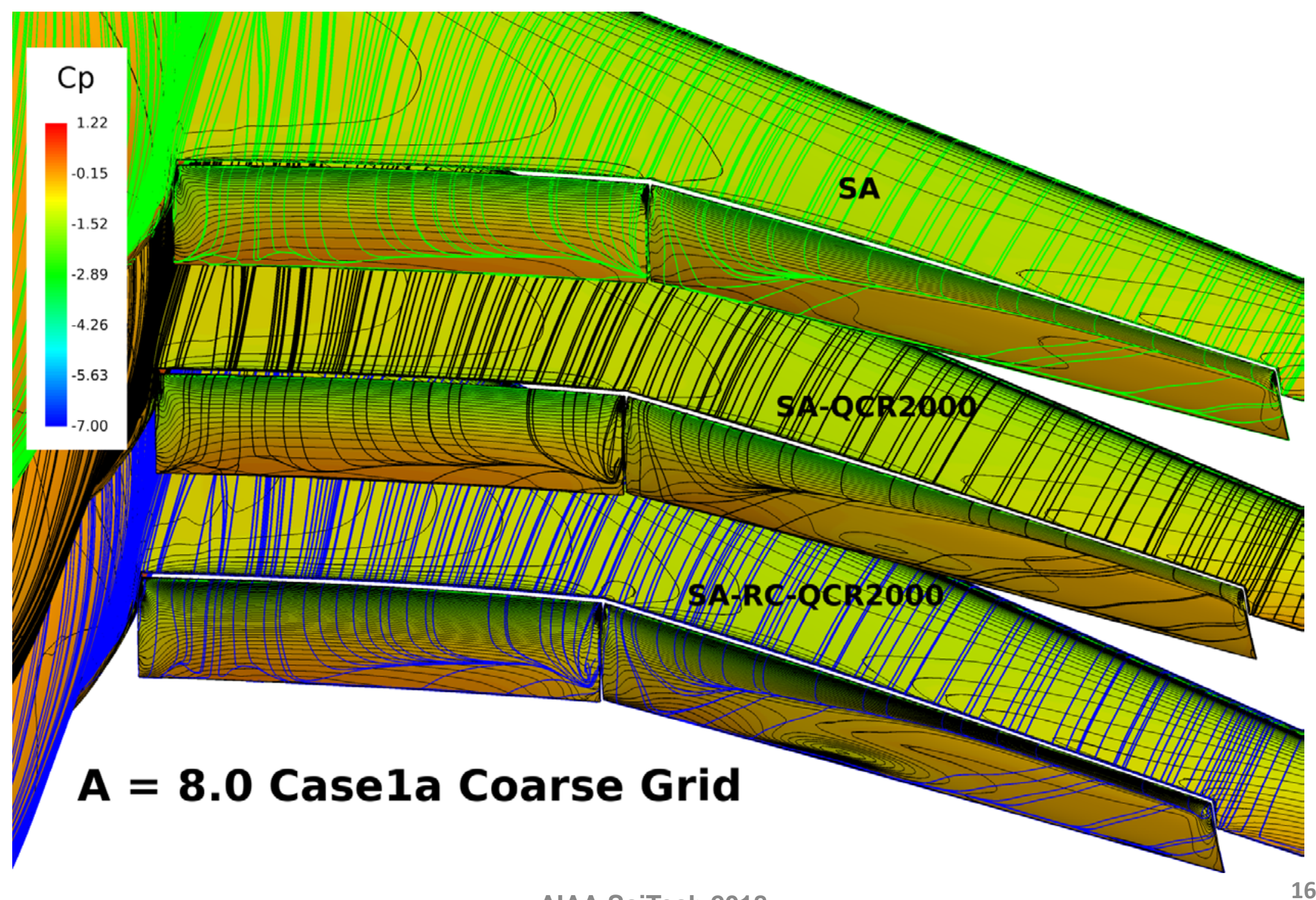




\section{Case 1: Grid Refinement Study}

- Lift
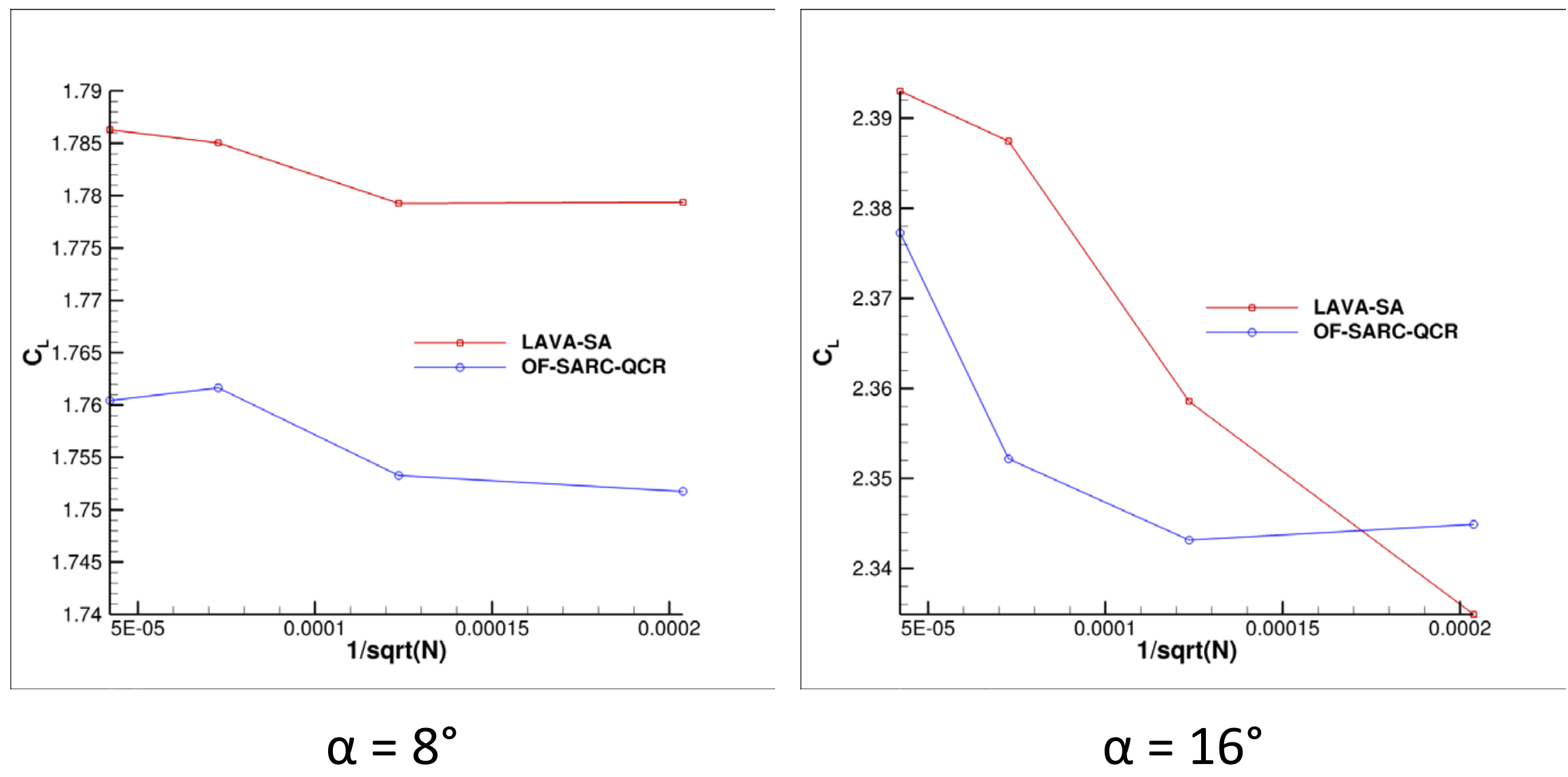

$$
\alpha=8^{\circ}
$$




\section{Case 1: Grid Refinement Study}

- Drag

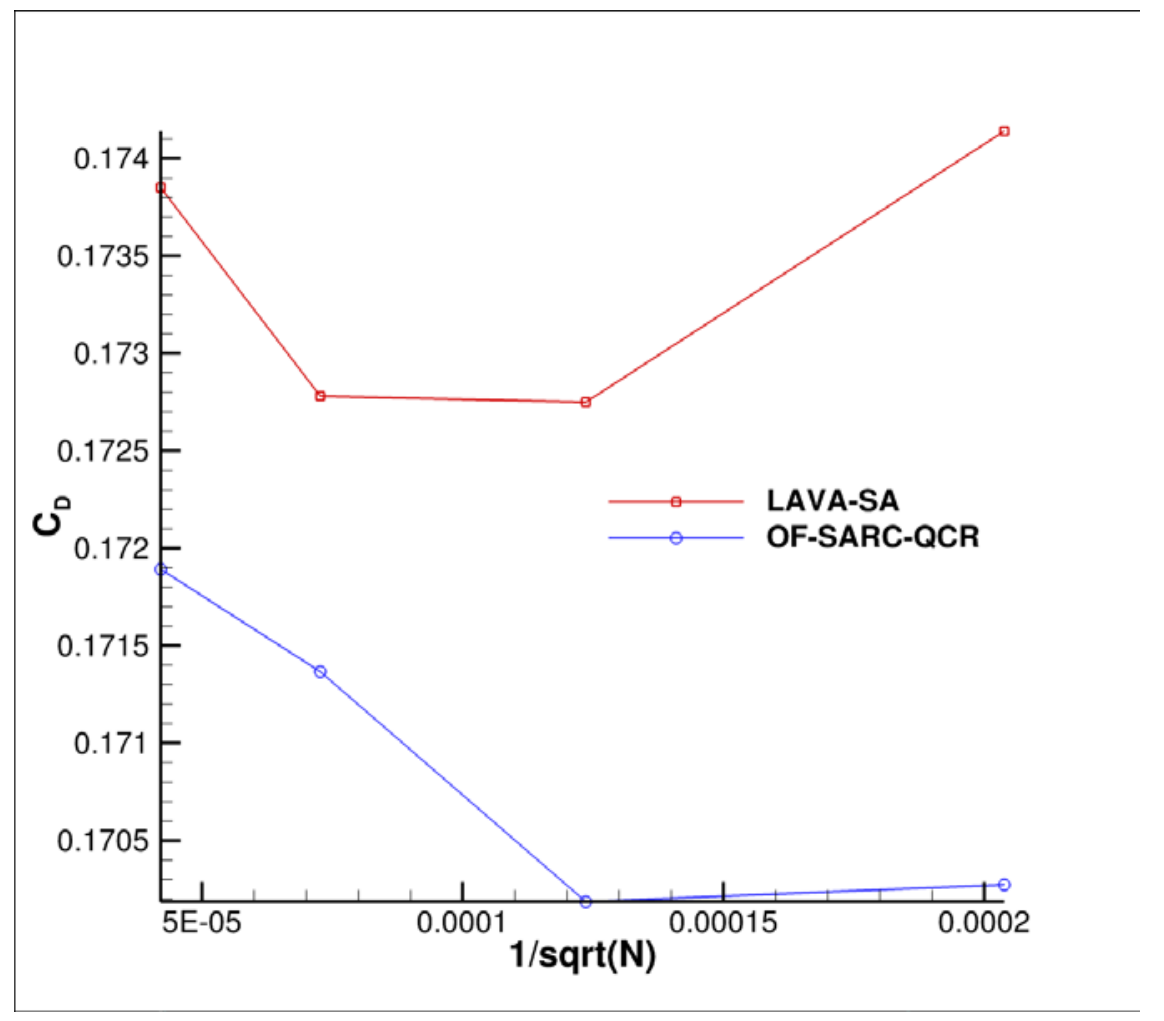

$$
\alpha=8^{\circ}
$$

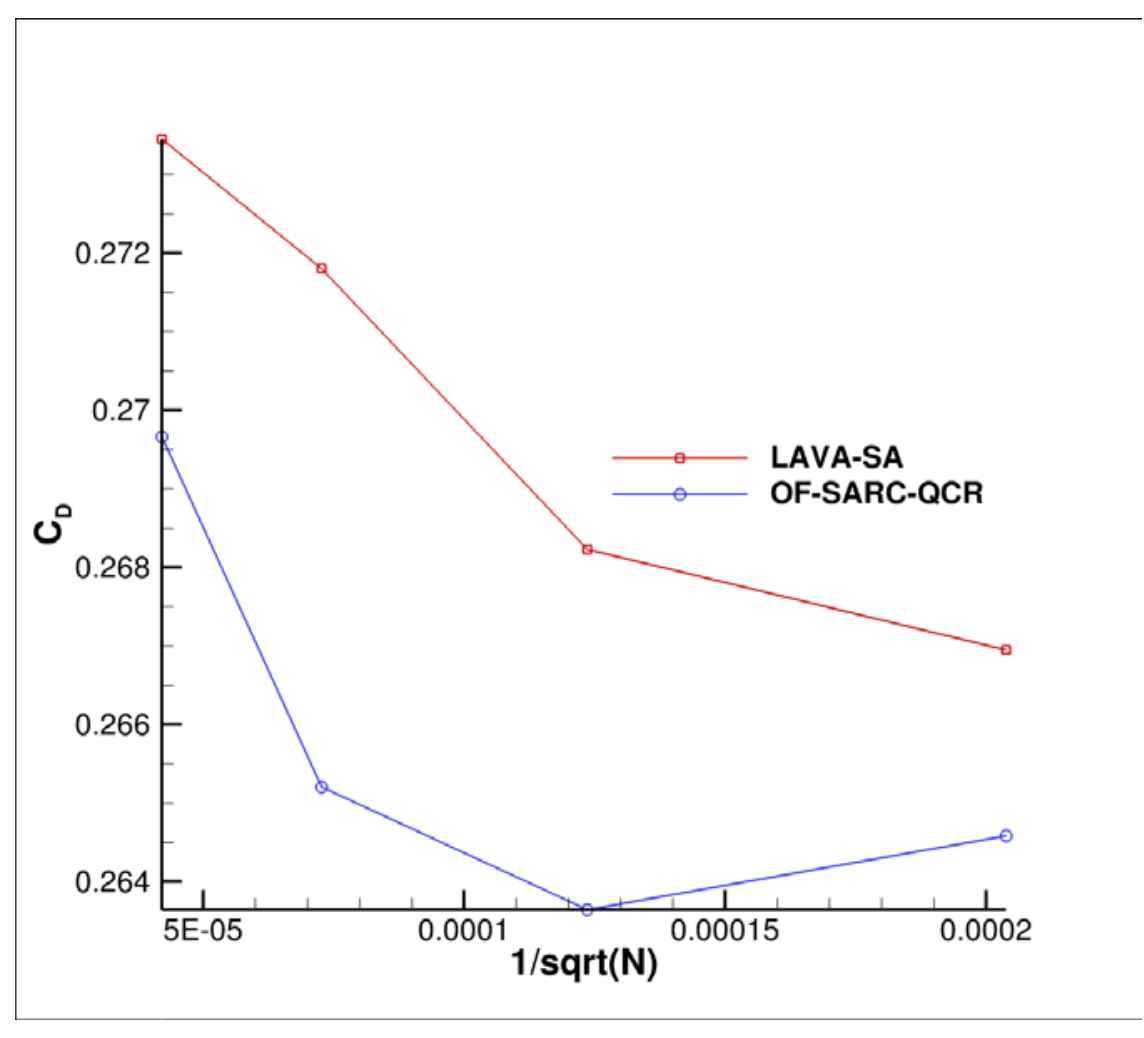

$$
\alpha=16^{\circ}
$$




\section{Case 1: Grid Refinement Study}

- Pitching Moment
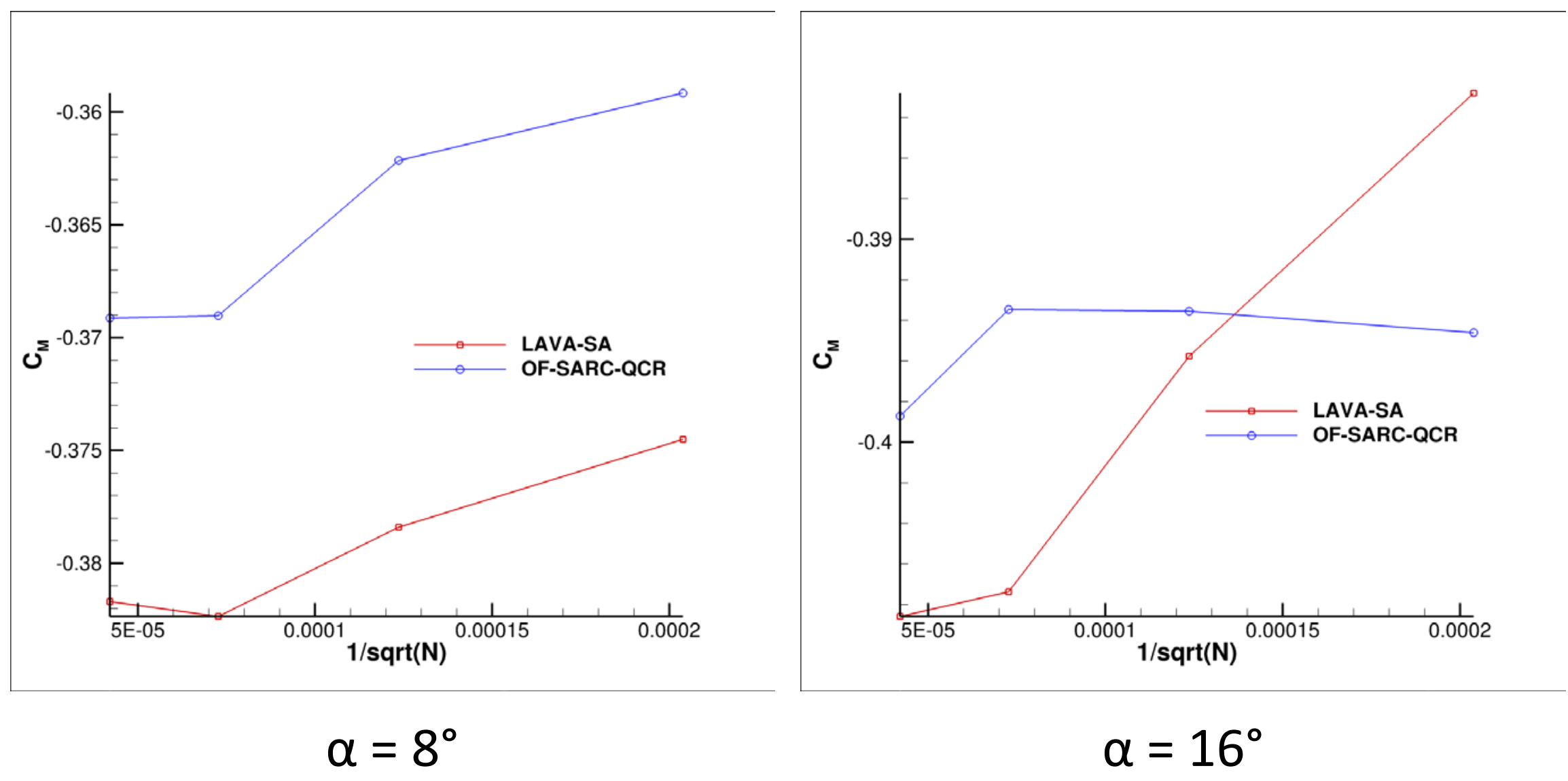

$$
\alpha=8^{\circ}
$$




\section{Case 1: Grid Refinement Study}

- Representative behavior $\left(\eta=0.151, \alpha=16^{\circ}\right)$
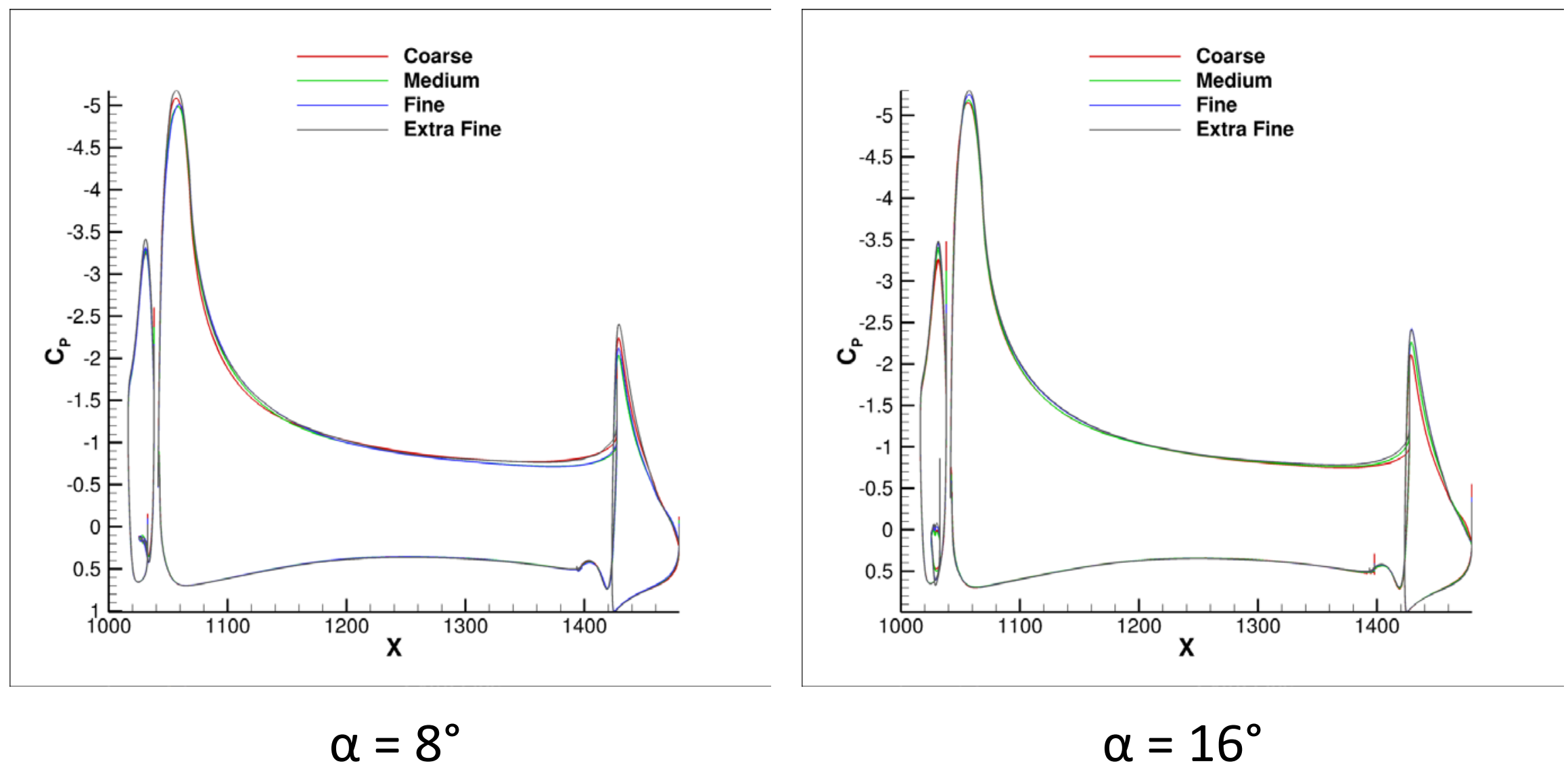


\section{Case 1: Effect of Flap Gap Seal}

- Gap seal reduces separation near the gap, but induces separation inboard

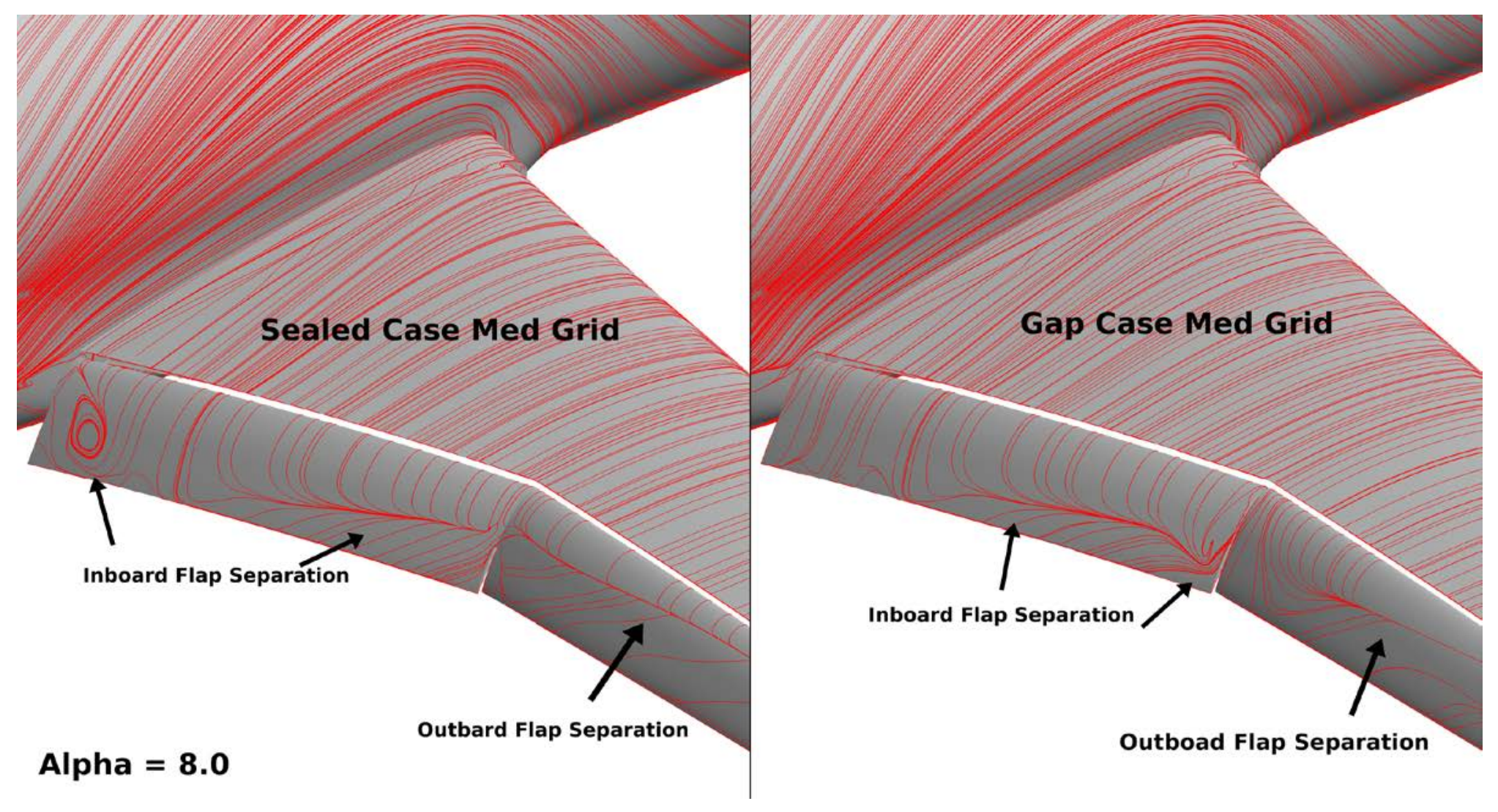




\section{Case 2: Nacelle/Pylon Off}

- Strong effect of turbulence/transition modeling

- Multiple possible solutions depending on initial condition

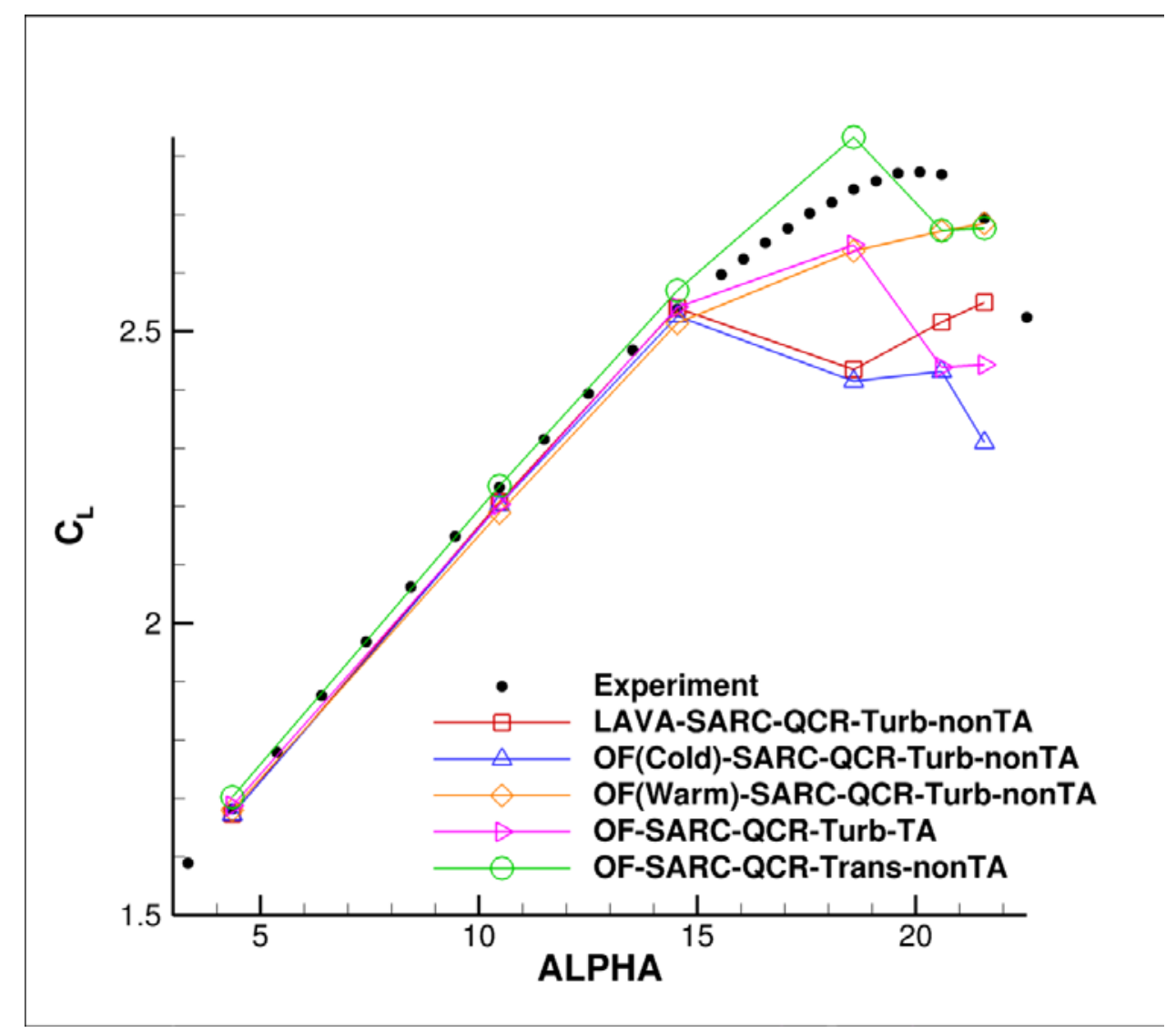




\section{Case 2: Nacelle/Pylon Off}

- Selected pressure distribution (4.36 deg)

Main element, $\eta=0.89$

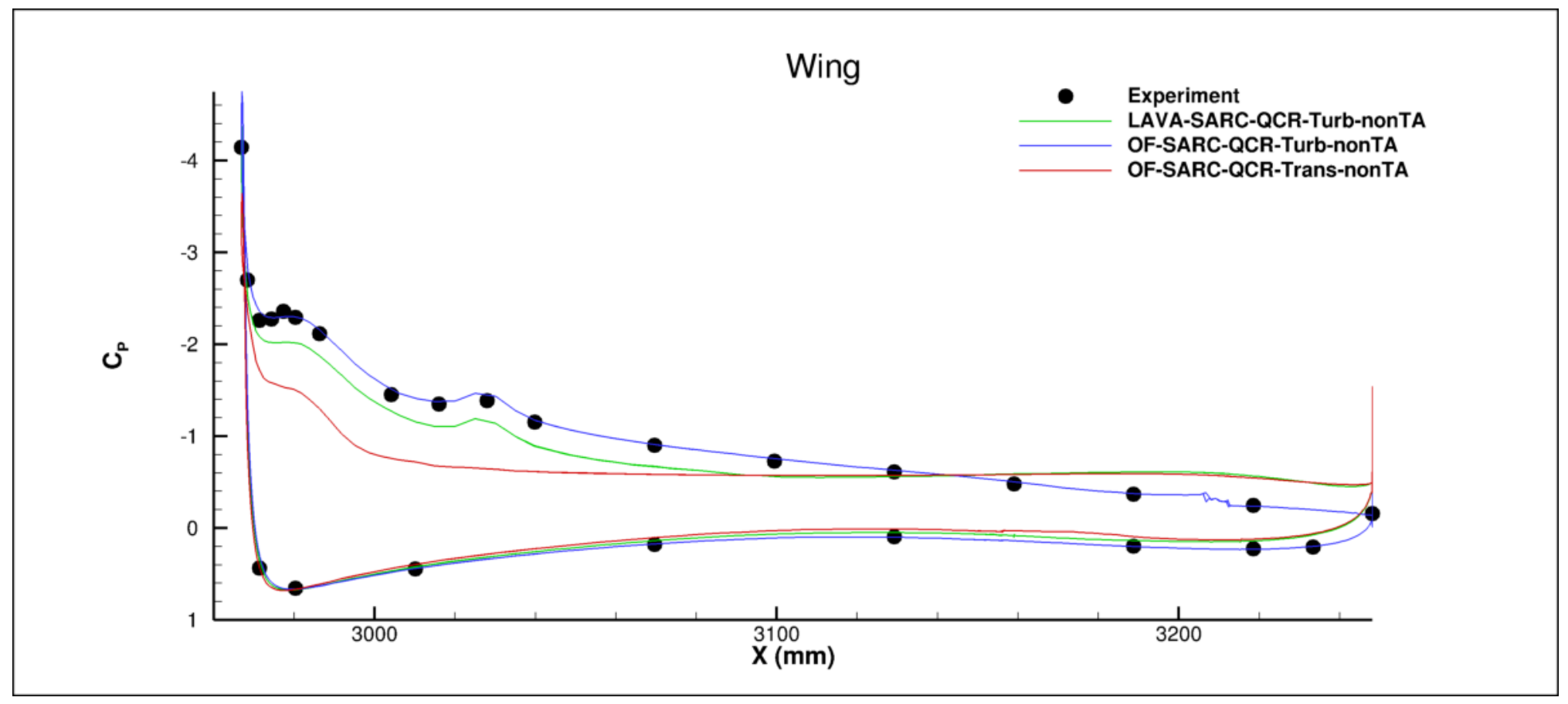




\section{Case 2: Nacelle/Pylon Off}

- Selected pressure distribution (18.58 deg)

Main element

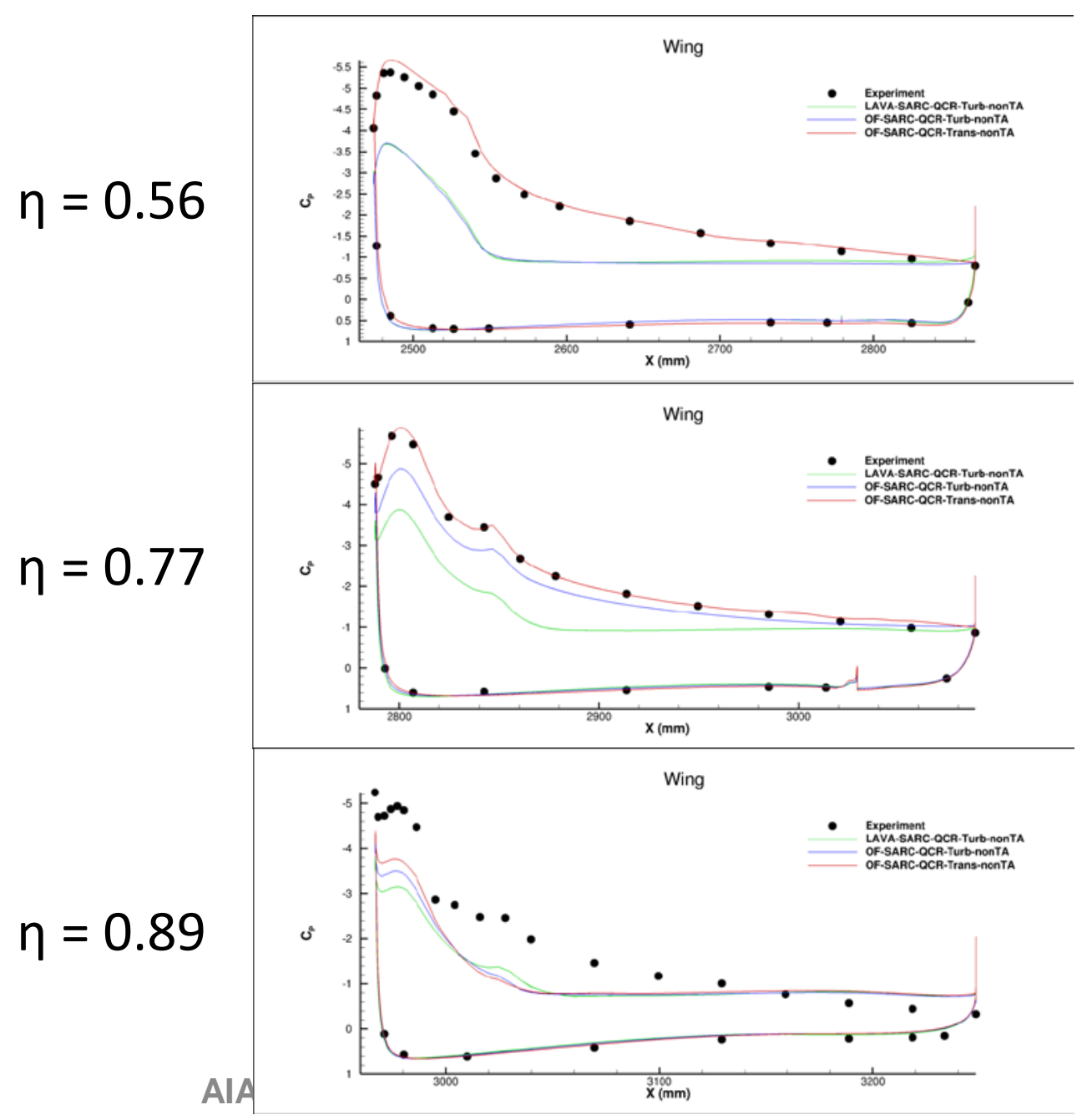




\section{Case 2: Nacelle/Pylon On}

- Strong effect of turbulence/transition modeling

- No evidence of multiple solutions

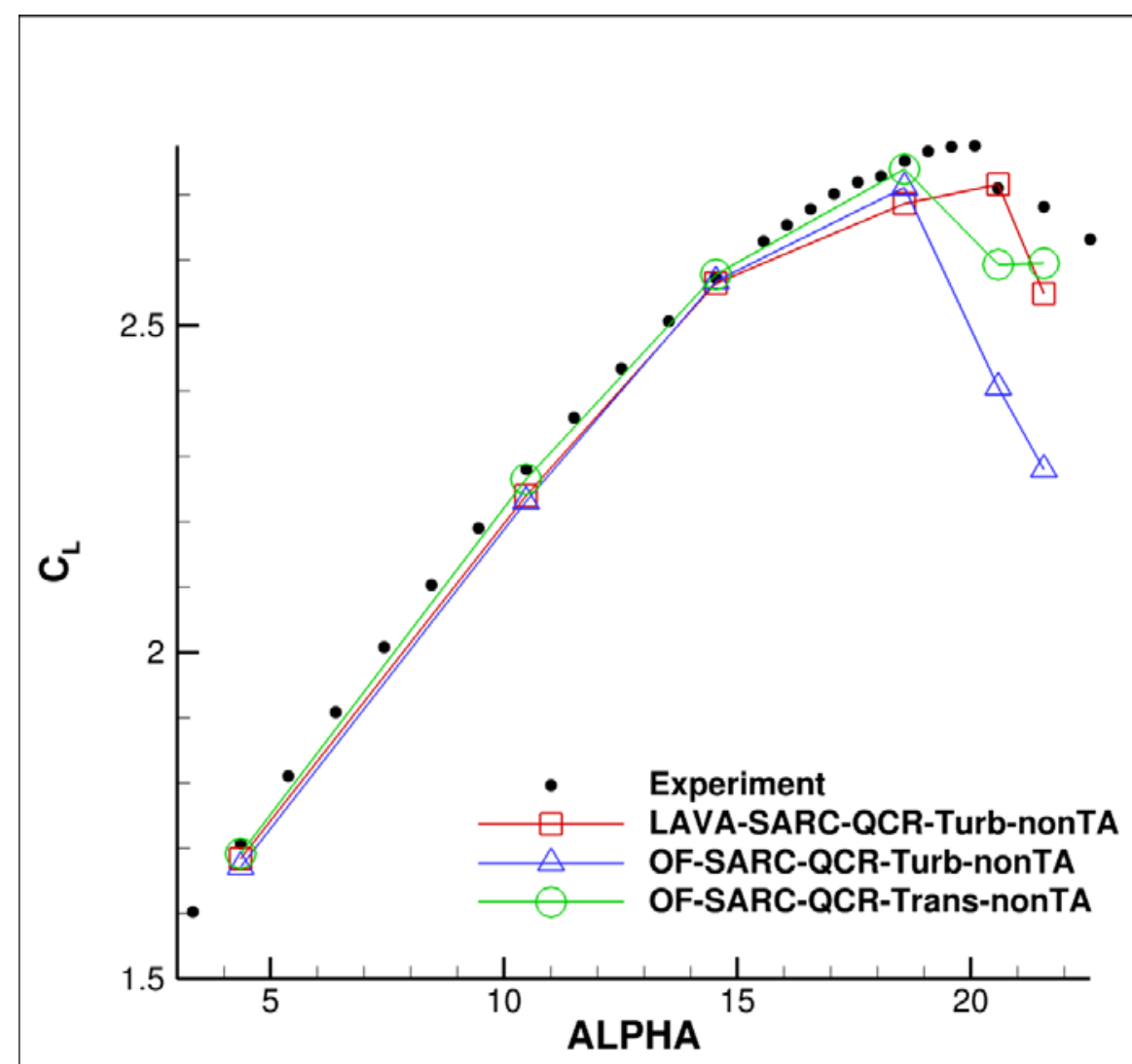




\section{Case 2: Nacelle/Pylon On}

- Surface flow patterns $\left(\alpha=18.58^{\circ}\right)$
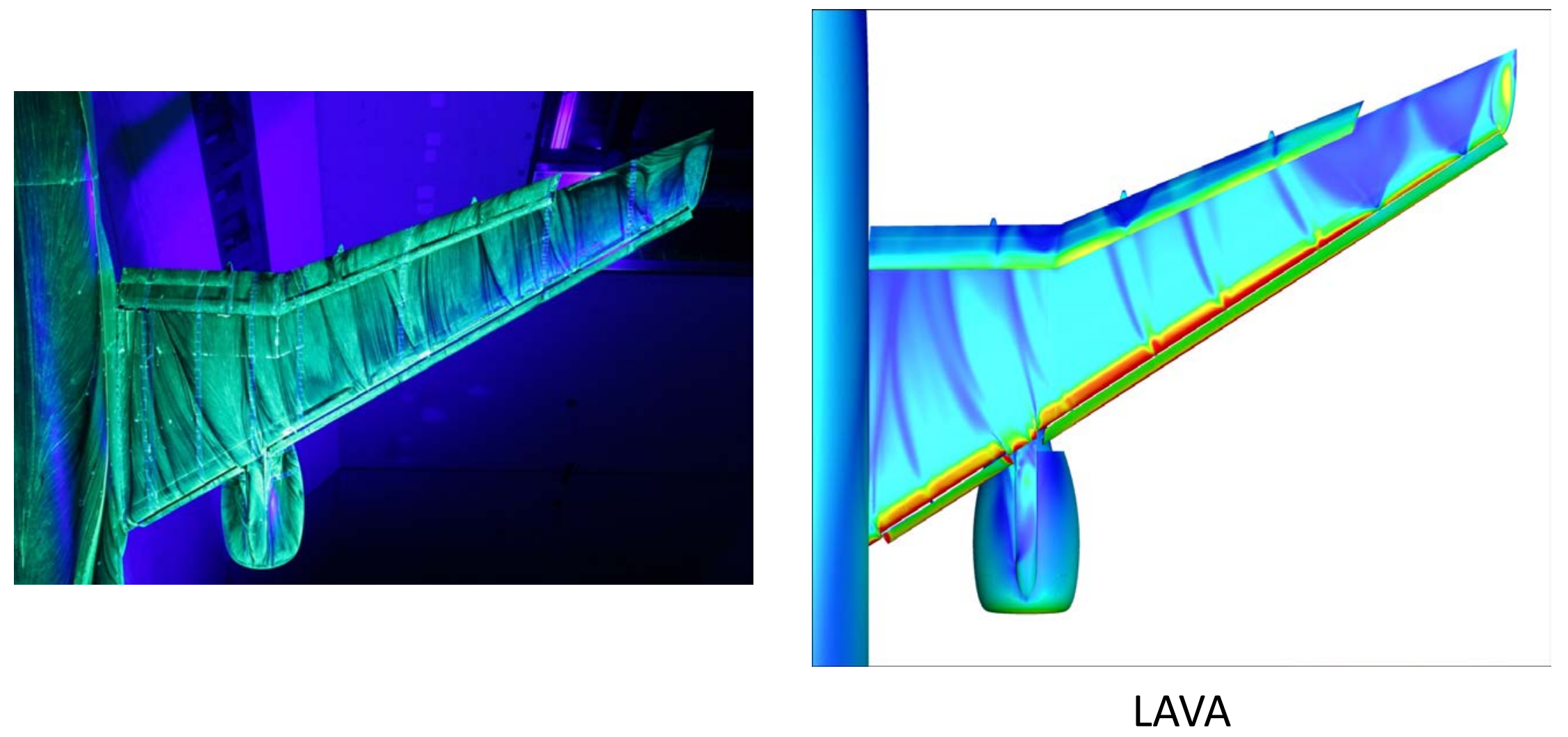


\section{Case 2: Nacelle/Pylon On}

- Surface flow patterns $\left(\alpha=18.58^{\circ}\right)$
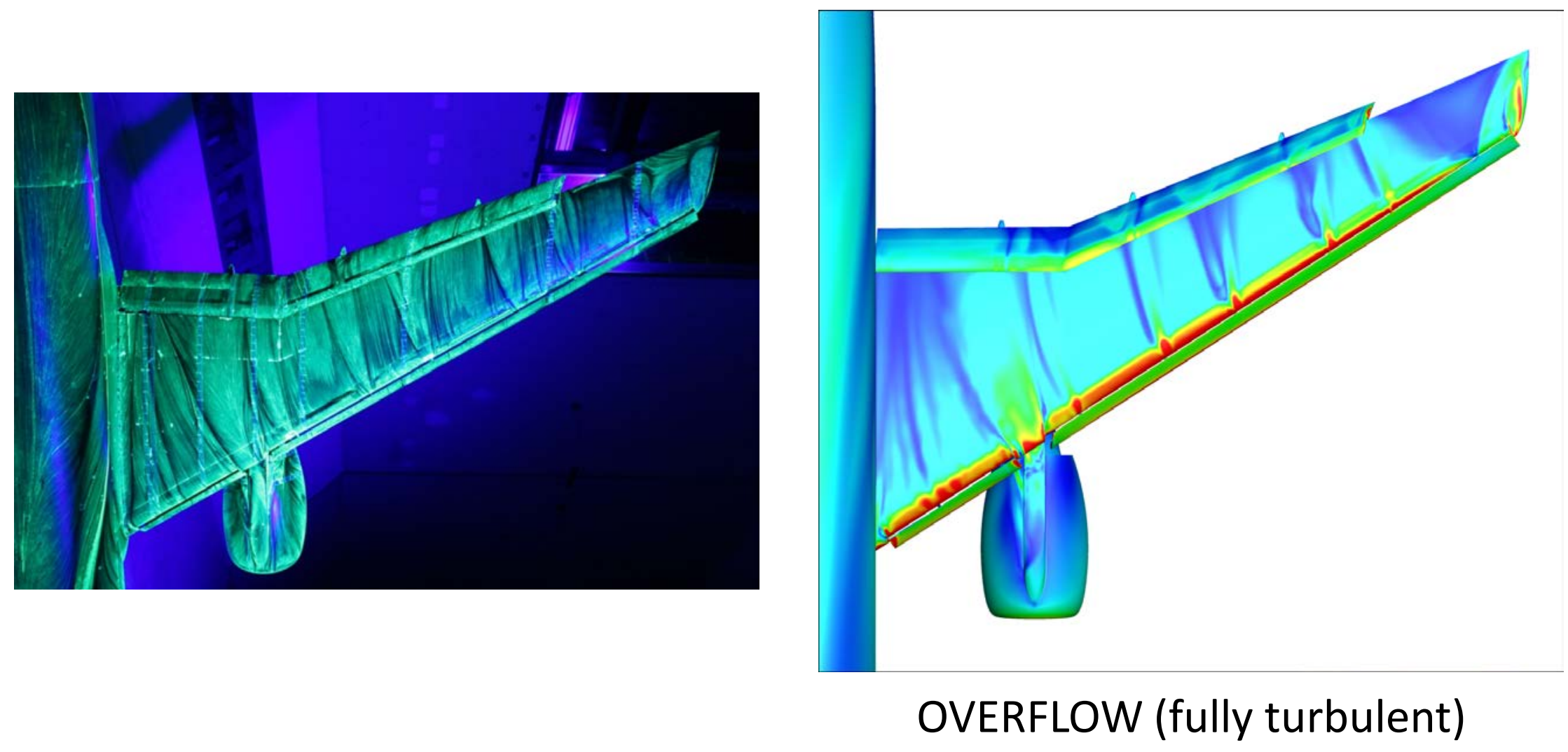


\section{Case 2: Nacelle/Pylon On}

- Surface flow patterns $\left(\alpha=18.58^{\circ}\right)$
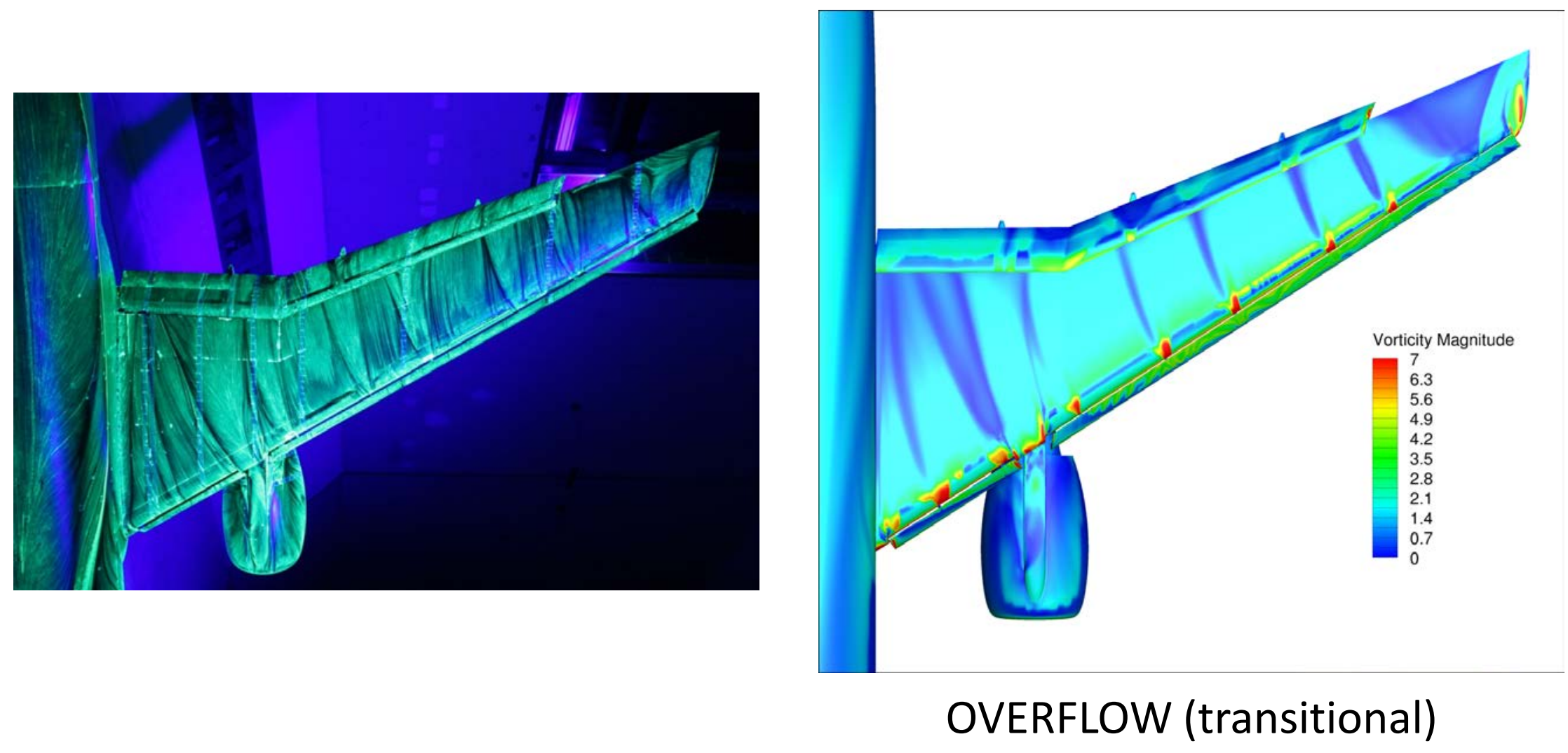


\section{Case 2: Nacelle/Pylon On}

- Transition patterns $\left(\alpha=18.58^{\circ}\right)$

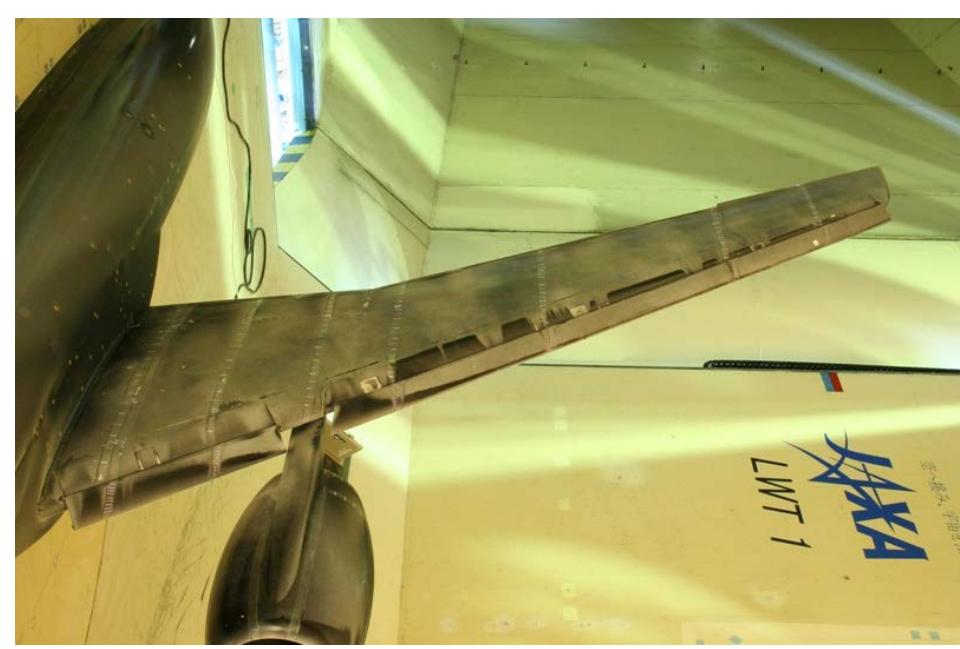

Experiment (China clay)

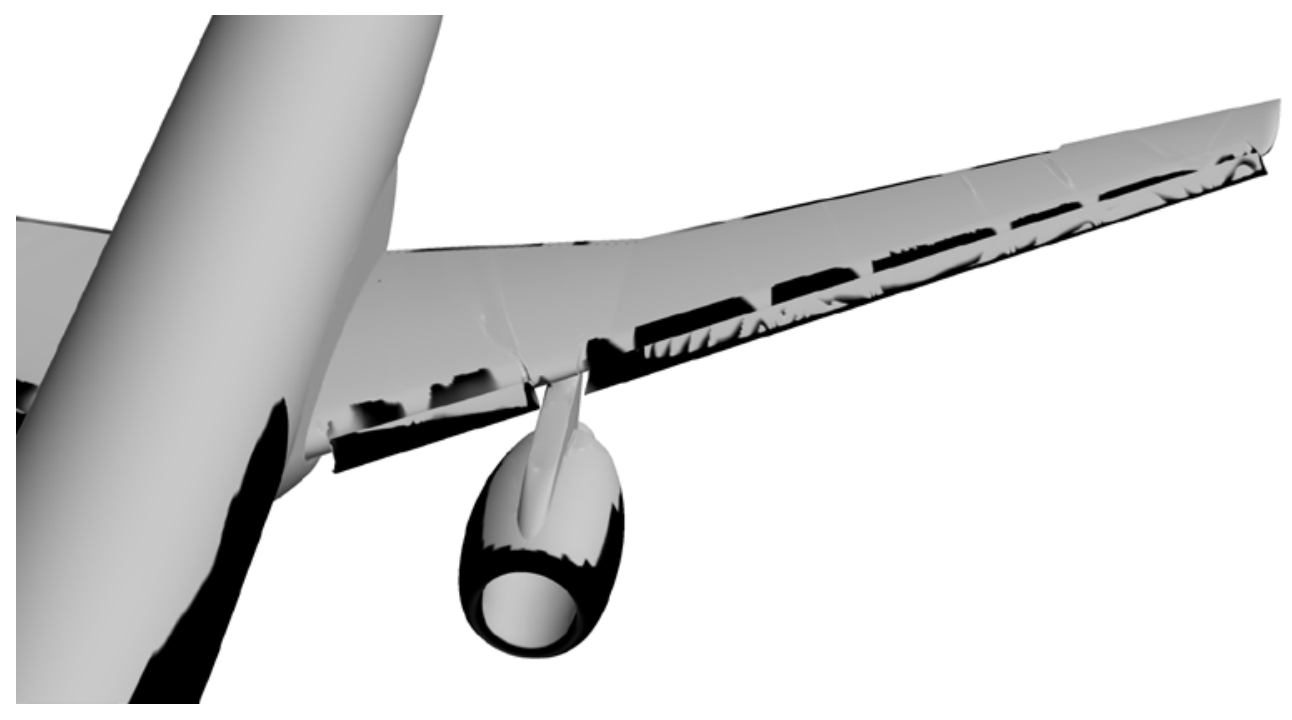

OVERFLOW (turbulent index) 


\section{Case 2: Nacelle/Pylon On}

- Transition patterns $\left(\alpha=18.58^{\circ}\right)$

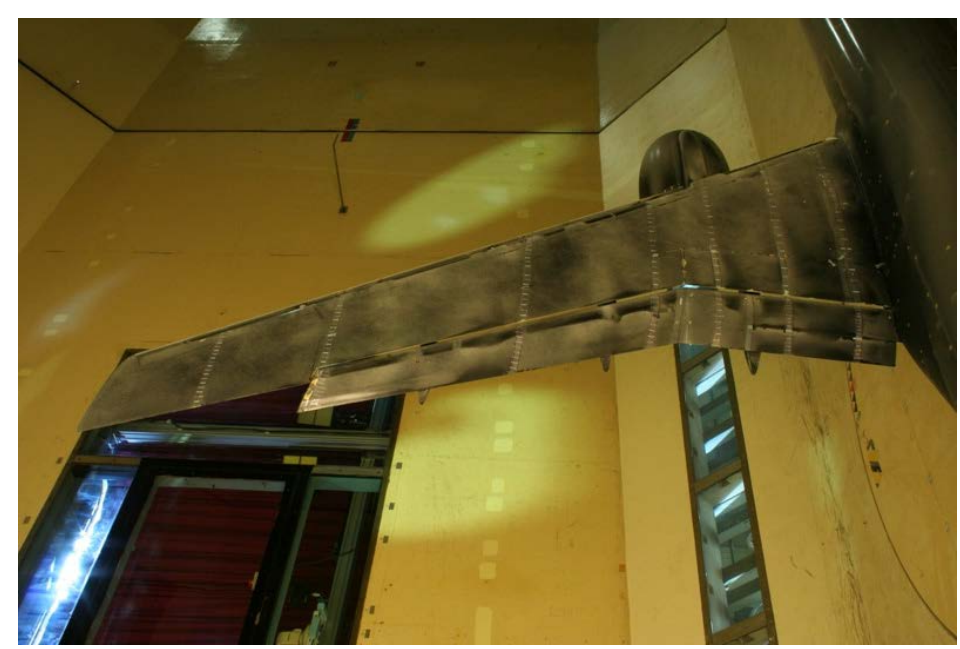

Experiment (China clay)

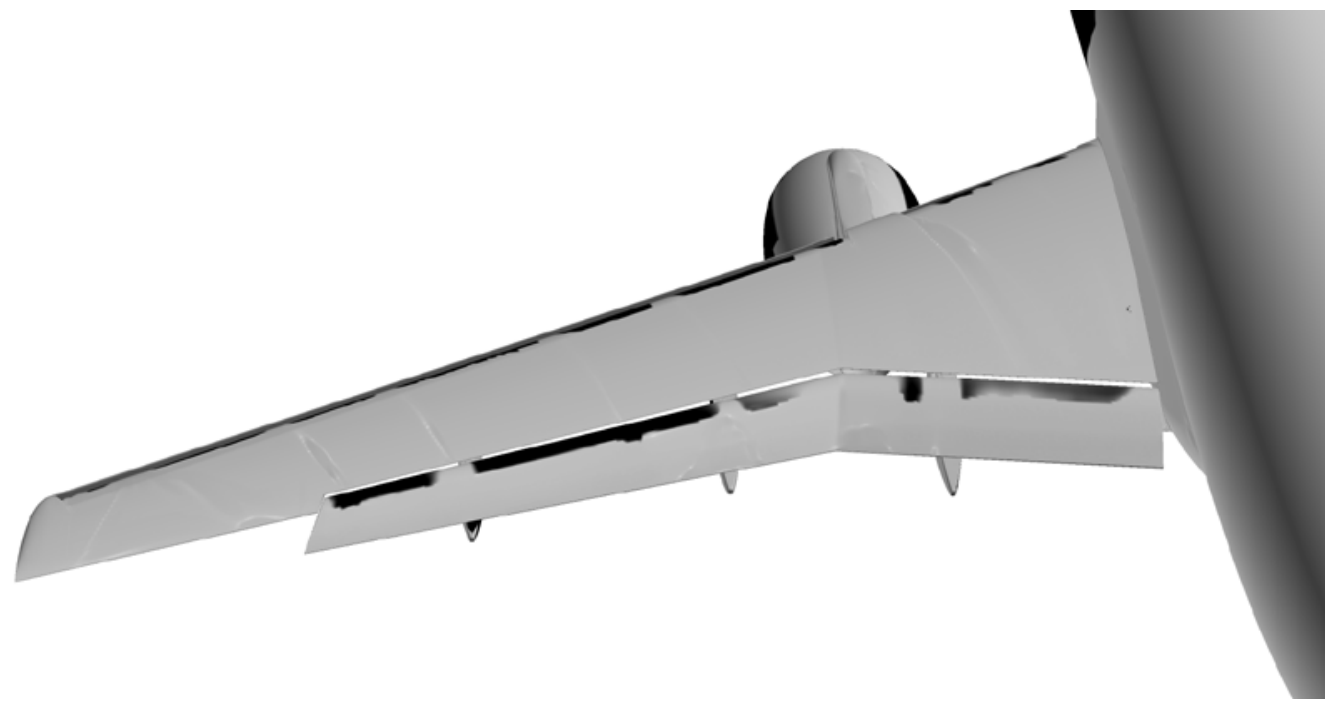

OVERFLOW (turbulent index) 


\section{Outline}

- Introduction

- Description of HiLiftPW-3 Geometries and Cases

- Computational Methodologies

- Results

- Conclusions

- Acknowledgments 


\section{Conclusions (HL-CRM)}

- Fully predictive, so no experimental data available for comparison

- Surface smoothness had an impact on surface pressure distributions

- Grid should be projected to smooth CAD rather than triangulated surfaces

- Use of QCR had a strong influence of flap separation patterns with the unsealed flap gap 


\section{Conclusions (JSM)}

- Evidence of multiple solutions observed for nacelle/pylon off

- "Warm" versus "cold" starts influenced final solution

- Time accurate results more consistent with warm starts

- Phenomenon not observed with nacelle/pylon on

- Excluding QCR had an impact, but not a consistent shift

- Nacelle/pylon off: Excluding QCR delays stall with AoA

- Nacelle/pylon on: Excluding QCR accelerates stall with AoA

- Transition modeling had an overall positive impact

- Better agreement in aerodynamic coefficients

- Predicted transition patterns consistent with experiment

- Not a panacea - separation patterns still have discrepancies 


\section{Outline}

- Introduction

- Description of HiLiftPW-3 Geometries and Cases

- Computational Methodologies

- Results

- Conclusion

- Acknowledgments 


\section{Acknowledgments}

- J.G. Coder thanks Cetin Kiris of NASA Ames Research Center for providing access to the NASA Advanced Supercomputing (NAS) Pleiades cluster 


$$
\sqrt{12}
$$

